\title{
Neck neuromuscular treatment for depressive disorders with cervical muscular tension or chronic neck pain -A pilot study
} \author{
Katsumi Nakamura ${ }^{2}$ \\ ${ }^{1}$ Tokyo Neuro Center, Tokyo, Japan \\ ${ }^{2}$ Yokakai Association, Fujimoto Hospital, Miyazaki, Japan \\ Email: ${ }^{*}$ ceo@fujimoto.or.jp
}

Takayoshi Matsui ${ }^{1}$, Toshiro Fujimoto ${ }^{2 *}$, Yuzo Endo ${ }^{2}$, Syuntaro Hojo ${ }^{1}$, Manabu Sakuda ${ }^{1}$, Syuji Kamano ${ }^{1}$, Kenichi Hirao ${ }^{1}$, Kayoko Matsumura ${ }^{1}$, Masaki Matsui ${ }^{1}$, Kouzou Takeuchi ${ }^{2}$,

Received 1 December 2011; revised 31 December 2011; accepted 10 January 2012

\begin{abstract}
BACKGROUND: An intimate relationship between depressive disorders and chronic pain is well known but often neglected. We studied patients with depressive disorders accompanying cervical muscular tension or neck pain. They also complaints of various physical, psychosomatic, and psychiatric signs such as anxiety and non-specific complaints related to autonomic imbalance. These complaints or symptoms are commonly resistant to pharmacological treatment, and thus we tried to treat these conditions by reducing their cervical muscular tension or pain. METHODS: We evaluated 30 complaints of 138 patients suffering from depressive disorders with cervical muscular abnormality (52 men and 86 women). The presence of the $\mathbf{3 0}$ complaints was evaluated as "positive" or "negative" before and during treatment. The necks of all patients were treated using low-frequency stimulation, micro-wave therapies, electric needle application, and acupuncture. RESULTS: Reducing cervical muscular tension remitted or cured the $\mathbf{3 0}$ complaints including depressive mood. The reduction of cervical muscle pain or tension initially ameliorated the depressive symptoms and anxiety, and subsequently improved their autonomic imbalances. Discriminant analysis of the first and second examinations correctly classified $95.3 \%$ of original grouped cases. CONCLUSION: Reducing cervical muscular tension is an effective treatment for patients suffering from depressive disorders with cervical neuromuscular pain or hardness.
\end{abstract}

Keywords: Depressive Disorders; Autonomic Nerve; Cervical Muscular Tension; Neck Pain; Anxiety

"Corresponding author.

\section{INTRODUCTION}

Many studies have shown an intimate relationship between depressive disorders and pain [1,2], but pain is an often-neglected symptom of depressive disorders [3]. Pain and tension in the musculoskeletal system are closely associated with emotion, a state mental condition, autonomic neurological manifestations [4-6], headache [7-13] and anxiety [14]. In addition, the remission of depressive symptoms due to the treatment of physical symptoms has been reported [15-17]. Musculoskeletal dysfunction of the neck is a contributing factor to the etiology of migraines and tension headaches $[18,19]$. However, these physical signs related to depressive disorders are often ignored because the widely-used DSM-IV does not include them as an integral part of the clinical picture of depressive disorders [20].

We have reported three cases of patients with depressive disorders accompanied by cervical tension or neck pain who showed a variety of subjective complaints of a physical and psychiatric nature [21]. In this study, we studied the 138 patients with these symptoms using the statistical method. The clinical pictures of these patients are variegated, and we classified them as 30 complaints or subjective and physical signs (depressive mood, neck pain or tension, headache, loss of interest, severe fatigability, generalized anxiety, vertigo, nausea and anorexia, sleep disturbance, visual difficulty, and autonomic disturbance such as unstable blood pressure, frequent sweating, pounding heart at rest, dry eye or excessive tears, mild fever, copious or absent saliva production) as shown in the Table 1. These patients are commonly resistant to pharmacologic treatments such as antidepressants, and their cervical tension is generally ignored. Although the patients in this study had undergone various examinations for such complaints, the cause was not identified, and no organic disease was revealed. They initially vis- 
ited an internist and then underwent examinations at otolaryngology, ophthalmology, gynecology, orthopedic, neurosurgery, and neurology clinics. However, no abnormality explaining the symptoms was identified, and the patients were finally referred to psychosomatic medicine or psychiatry clinics, at which they were diagnosed with conditions such as autonomic imbalance, menopausal syndrome, chronic fatigue syndrome, fibromyalgia, vertigo, mood disorders, anxiety disorders, panic disorders, somatoform disorders and adjustment disorders and were treated with tranquilizers, antidepressants, analgesics, and sleeping drugs. Nonetheless, their cervical muscle tension and pain were not examined or treated, despite causing severe impairment.

We treated the patients with depressive disorders accompanying cervical muscular tension or neck pain and the above-mentioned complaints or symptoms, using methods of physiotherapy such as low-frequency stimulation, microwave therapies, electric needle application, and acupuncture methods to reduce their cervical tension or pain. Almost all of the 30 complaints were remitted during the course of the treatment. The objective of this study is to report efficacy of treatment to reduce cervical muscular tension or neck pain patients accompanied by depressive disorders.

\section{MATERIALS AND METHODS}

\subsection{Study Population}

The subjects were 138 patients who were admitted to Matsui Hospital and underwent treatment for depressive disorders with cervical muscular abnormality. The inclusion criteria were the presence of cervical muscle tension, neck pain, autonomic imbalance, and psychiatric symptoms such as depressive disorders and anxiety. The mean age was 42.8 years. There were 52 males (mean age: 38.8 years, S.D. 13.5 ) and 86 females (mean age: 45.3 , S.D. 16.1). Their complaints of physical, psychosomatic, and psychiatric signs were classified as items of 1 - 30 and are shown in Table 1. In the index of symptoms (Table 1), the presence of the 30 symptoms was evaluated as "yes" or "no". All patients underwent cervical X-ray radiography, CT, MRI, MRA, EEG, and a balance test, but no organic abnormality was found, including in the neck, and all findings were within normal ranges.

\subsection{Treatments}

We used various physiotherapies of western and Oriental medicine to reduce cervical muscle tension: a hot pack (Nihon Medix) for 15 minutes, low-frequency silver spike point (SSP) stimulation therapy (Nihon Medix) for 10 min, interferential therapy utilizing low-frequency stimulation (Nihon Medix) for $10 \mathrm{~min}$, microwave therapy (Nihon Medix) for $10 \mathrm{~min}$, electric needle application (Ze- niryoki), and acupuncture. For drug therapy, mainly antipsychotic vitamins were administered. Oral antidepressant medication started before visiting our hospital was maintained at the time of treatment initiation, depending on the symptoms. The dosage was reduced and the administration was finally discontinued as the effect of treatment appeared. The presence and absence (two categories) of abnormal muscle tonus and pain were investigated at a total of 34 points on the bilateral sides.

\subsection{Statistical Analysis Using Chi-Square Test}

The 30 symptoms on the list of subjective symptoms were evaluated before and after treatment. We counted the number of these items present in the first and second examinations. We conducted a chi-square test and obtained improved numbers for items 1 - 30 of the subjective symptoms, showing an association between the first and second examinations using SPSS software. Items 1 to 19 are related to autonomic disturbances, and items 20 to 30 are related to depressive disorders and anxiety. Items 22, 24 and 26 are related to three major symptoms of the depressive disorders with cervical neuromuscular pain or hardness. Items 25, 27 and 28 are related to minor symptoms of this type of depressive disorders. To examine the correlation between autonomic dysfunctions and depressive disorders and anxiety, we calculated coefficients of correlation between items related to autonomic disorders (items 1 - 19). We also conducted discriminant analysis of the first and second examinations for the 30 items.

\section{RESULTS}

The mean duration of cervical muscle relaxation treatment was 91.1 days. Depressive and anxiety states improved as autonomic dysfunctions improved during the course of treatment to reduce muscular tension. All patients showed improvements in items 1 - 30 after cervical muscle relaxation treatment, as shown in Table $\mathbf{1}$. The Index of symptoms showed complaints in 129 patients for items 2, 3, and 21; 119 for item $1 ; 117$ for item 14; 115 for item 20; 108 for item 25; 104 for item 28; 103 for item 24; 102 for item 22; 100 for item 5; 99 for item 8; 98 for item 29; 91 for item 27; 90 for item 23; 81 for item 26; 80 for item 6; 78 for item 11; 67 for items 10 and 16; 66 for item 19; 65 for items 13 and 15; 61 for item 12; 58 for item 4; 54 for item 7; 52 for item 9; 48 for item 30; 43 for item 18; and 31 for item 17. The cure rates of the index of symptoms (ratios of first to second examinations) were $98.4 \%$ for item 12 in all cases, $98.0 \%$ for item 22, $97.2 \%$ for item $25,97.1 \%$ for items 24 and 28, 95.1\% for item 26, 93.9\% for item 19, 93.4\% for item $27,90.7 \%$ for item $7,90.4 \%$ for item $9,90.3 \%$ for item $17,89.7 \%$ for item $4,89.6 \%$ for item $16,88.4 \%$ for item $18,88.1 \%$ for item $10,87.8 \%$ for item 20 , 
$87.7 \%$ for items 13 and $15,87.5 \%$ for item $30,86.3 \%$ for item $14,84.4 \%$ for item $23,82.9 \%$ for item 21 , $81.6 \%$ for item $29,80.0 \%$ for item $5,77.3 \%$ for item 1 , $74.4 \%$ for item $11,72.7 \%$ for item $8,72.5 \%$ for item 6 , $55.8 \%$ for item 3 , and $51.9 \%$ for item 2 . The chi-square test showed improved numbers for items 1 - 30 of the index of symptoms, showing associations between the first and second examinations (Table 2). Items 20 - 30, which are related to depressive symptoms and anxiety, were significantly correlated to items $1-19$, which are

Table 1. Index of symptoms of 30 complaints of physical, psychosomatic, and psychiatric signs and recovery rates (Total 138 Cases).

\begin{tabular}{|c|c|c|c|c|}
\hline & Symptoms & First examination & Second examination & Recovery rate (\%) \\
\hline 1 & Headache or heavy headedness & 119 & 27 & 77.3 \\
\hline 2 & Neck pain or tension & 129 & 62 & 51.9 \\
\hline 3 & Stiff shoulders & 129 & 57 & 55.8 \\
\hline 4 & Frequently catching a cold & 58 & 6 & 89.7 \\
\hline 5 & Light headedness or vertigo & 100 & 20 & 80 \\
\hline 6 & Feeling unstable while walking or standing & 80 & 22 & 72.5 \\
\hline 7 & Nausea, anorexia & 54 & 5 & 90.7 \\
\hline 8 & $\begin{array}{l}\text { Difficulty in falling asleep and intermittent awakening at } \\
\text { night }\end{array}$ & 99 & 27 & 72.7 \\
\hline 9 & Unstable blood pressure & 52 & 5 & 90.4 \\
\hline 10 & $\begin{array}{l}\text { Difficulty remaining in a warm place for a prolonged } \\
\text { period (abnormal body temperature control) }\end{array}$ & 67 & 8 & 88.1 \\
\hline 11 & Frequent sweating & 78 & 20 & 74.4 \\
\hline 12 & Pounding heart at rest & 61 & 1 & 98.4 \\
\hline 13 & Visual difficulty, blurry vision & 65 & 8 & 87.7 \\
\hline 14 & Easily tired or painful eyes & 117 & 16 & 86.3 \\
\hline 15 & Over-sensitivity to light or difficulty in keeping eyes open & 65 & 8 & 87.7 \\
\hline 16 & Dry eyes or excessive tears & 67 & 7 & 89.6 \\
\hline 17 & Saliva production copious or absent & 31 & 3 & 90.3 \\
\hline 18 & Mild fever (at $37^{\circ} \mathrm{C}$, occasionally exceeds $38.0^{\circ} \mathrm{C}$ ) & 43 & 5 & 88.4 \\
\hline 19 & $\begin{array}{l}\text { Frequent diarrhea (gastrointestinal symptoms, such as } \\
\text { abdominal pain) }\end{array}$ & 66 & 4 & 93.9 \\
\hline 20 & Habit of lying down at daytime & 115 & 14 & 87.8 \\
\hline 21 & Severe fatigability & 129 & 22 & 82.9 \\
\hline 22 & Loss of interest in all activities or motivation & 102 & 2 & 98 \\
\hline 23 & Feeling unwell during bad weather or the day before & 90 & 14 & 84.4 \\
\hline 24 & Depressive mood or feeling down & 103 & 3 & 97.1 \\
\hline 25 & Loss of concentration or attention & 108 & 3 & 97.2 \\
\hline 26 & Generalized anxiety & 81 & 4 & 95.1 \\
\hline 27 & Irritation or frustration & 91 & 6 & 93.4 \\
\hline 28 & $\begin{array}{l}\text { Impatience, difficulty in working or studying for long } \\
\text { hours }\end{array}$ & 104 & 3 & 97.1 \\
\hline 29 & Hot flushes, cold or numb hands or legs & 98 & 18 & 81.6 \\
\hline 30 & Pain, pressure, or numbness in the chest & 48 & 6 & 87.5 \\
\hline
\end{tabular}




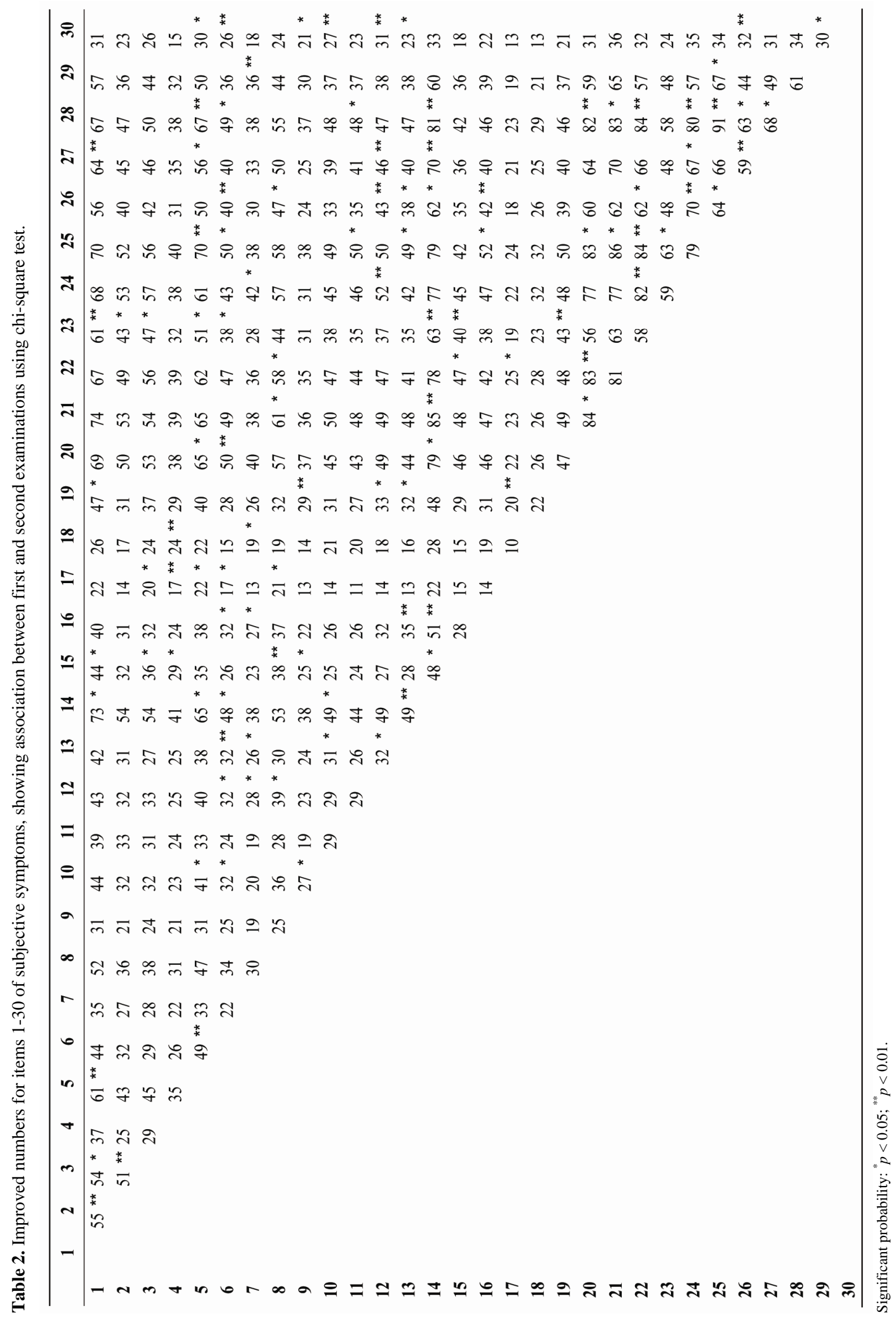


related to autonomic dysfunction in the index of symptoms ( $p=6.29 \mathrm{E}-11)$. Discriminant analysis of the first and second examinations correctly classified $95.3 \%$ of the originally grouped cases.

\section{DISCUSSION}

We studied patients with depressive disorders accompanied by cervical muscle tension or neck pain and a large variety of complaints of physical, psychosomatic signs. The 30 items of the symptoms shown on Table 1 were cured by neck muscle treatment using therapies including low-frequency stimulation, microwave, electric needle application, and acupuncture. Discriminant analysis of the first and second examinations for the 30 items correctly classified $95.3 \%$ of originally grouped cases. This finding showed that reducing cervical muscular tension is an effective treatment for patients suffering depressive disorders with cervical neuromuscular pain or hardness.

Our dichotomous method had following limitations: depressive symptoms and other symptoms were collectively handled; the degree of these symptoms was not evaluated; the type of headache was not classified. However, this method widely evaluated psychiatric and physical symptoms without being weighted by these classifications, allowing psychiatric and physical symptoms to be objectively surveyed 2-dimensionally on the same plane. This facilitated understanding of the relationships among depressive symptoms and anxiety, autonomic imbalances, cervical muscular tension, and neck pain, while avoiding separation of psychiatric/physical symptoms.

Comparing the scores in the first examination before cervical muscle treatment with those of the second examination after a mean of 91.1 days of treatment for the 30 subjective symptoms shown on Table 1 showed a marked improvement of each complaint (Table 2). The following items showed higher recovery rates: loss of interest or motivation (item 22), loss of concentration or attention (item 25), depressive mood or feeling down (item 24), impatience, difficulty in working or studying for long hours (item 28), generalized anxiety (item 26), and irritation or frustration (item 27). There were also recoveries in items related to autonomic imbalances: pounding heart at rest (item 12), frequent diarrhea (item 19), nausea (item 7), unstable blood pressure (item 9), and copious or absent saliva production (item 17). These results showed that relaxation therapy aimed at reducing cervical muscular tension is effective at treating depressive disorders, anxiety, and symptoms of autonomic imbalance. On the other hand, headache or heavy headedness (item 1), neck pain or tension (item 2), and stiff shoulder [3] showed the comparatively lower recovery rates of $51.9 \%-77.3 \%$. One of the reasons is that cervical muscle tension and neck pain were generally more treatment resistant and tended to persist although they did improve, and physiotherapy was not sufficient to cure radical causes of muscular tension or pain resulting from organic lesions. Even if the physiotherapy for neck tenderness or pain was insufficient, it was effective in reducing subjective symptoms related to depressive disorders and autonomic disturbances, probably because it had some effect on the sympathetic or parasympathetic systems in the cervical region. Another reason was related to our dichotomous evaluation method. Although neck pain or tension and shoulder stiffness were relatively improved in most patients after treatment, they answered yes to residual neck pain or tension and shoulder stiffness when the symptoms were not completely recovered.

It was noted that depressive disorders and anxiety were also improved as autonomic imbalance improved. Chisquare tests of the scores for 30 items of the subjective symptoms in the first and second examinations (Table 2) showed many significant positive correlations among the 30 items. Depressive mood or feeling down (item 24) showed a significant correlation at the $p=0.01$ level with pounding heart at rest (item 12). Generalized anxiety (item 26) showed a significant correlation with feeling unstable while walking or standing (item 6), pounding heart at rest (item 12), and dry eyes or excessive tears (item 16). These findings suggested that anxiety is also positively correlated with autonomic imbalances. The correlations between overall autonomic imbalance (items 1 to 19) and overall depressive mood and anxiety (items 20 to 30) were significantly positive (Pearson's correlation index: $r=0.520, p=6.3 \mathrm{E}-11$ ) in the first examination, and significant correlations were also found in the second examination (Pearson's correlation index: $r=$ $0.577, p=3.3 \mathrm{E}-16)$. However, the improvement of autonomic neurological manifestations was not as marked as that of depressive mood and anxiety. The cure rates for feeling unstable while walking or standing (item 6), difficulty in falling asleep and intermittent awakening at night (item 8), and sweating easily (item 11) were lower at $72.5 \%, 72.7 \%$, and $74.4 \%$, respectively. This suggested that the reduction of cervical muscle tension initially improved the depressive mood and anxiety, and subsequently improved autonomic imbalances.

Antidepressants prescribed by other hospitals at the time of admission to our hospital were discontinued at a relatively early phase, if possible, or the dosage was gradually reduced as the symptoms remitted over several weeks. However, discontinuation was not possible in eight of the 138 patients $(0.06 \%)$, even though their cervical muscle tension and autonomic imbalances were relatively remitted and dose reduction was possible. These eight patients complained feelings of worthlessness or inappropriate guilt, which may have been delusional, every day. This finding suggested that depressive disorders 
with cervical tenderness or neck pain were different from major depression with feelings of excessive or inappropriate guilt or worthlessness. In addition, almost all patients of this study experienced a period with strong suicidal ideation. It was reported a higher rate of suicide completion for chronic pain patients [22], and the suicidal ideation of our patients may be related to this report.

Recently, it was reported that many patients in a mild depression with cervical tenderness or pain visit outpatient clinics. Physical concerns are risk factors for the development of depression longitudinally [23]. Physical symptoms in depression were required for specific physiotherapeutic treatment for patients with moderate to severe depression [16]. Work-related physical and psychosocial factors, as well as several individual risk factors, are associated with subjective pain and clinical signs in the neck and shoulders [24]. We presume that there are significant populations in a technetronic society who suffer an overload of the cervical muscles due to computerbased work combined with depressive disorders and anxiety, pharmacological treatment resistance, and autonomic imbalances whose cervical tension and pain are disregarded.

\section{CONCLUSION}

This study demonstrated that reducing cervical muscular tension is an effective treatment for patients suffering from depressive disorders with cervical neuromuscular pain or hardness accompanied by complaints of insomnia and anxiety, and autonomic imbalances. This suggested that cervical muscular tension or neck pain is closely related to depressive disorders, anxiety, and autonomic imbalances, giving a new perspective on the scientific analysis of body-mind correlations and therapeutic effects in the neck.

\section{REFERENCES}

[1] Currie, S.R. and Wang, J. (2004) Chronic back pain and major depression in the general Canadian population. Pain, 107, 54-60. doi:10.1016/j.pain.2003.09.015

[2] Lin, E.H.B., Tang, L., Katon, W., Hegel, M.T., Sullivan, M.D. and Unützer, J. (2006) Arthritis pain and disability: Response to collaborative depression care. General Hospital Psychiatry, 28, 482-486. doi:10.1016/j.genhosppsych.2006.08.006

[3] Stansfeld, S. (2006) Chronic pain, depressive disorder, and the role of work. Journal of Psychosomatic Research, 61, 661-662. doi:10.1016/j.jpsychores.2006.06.010

[4] Hare, M. (1985) The physical problems of depressive illness. Physiotherapy, 71, 258-261.

[5] Friis, S., Bunkan, B.H., Ljunggren, A.E., Moen, O. and Opjordsmoen, S. (1998) What are the basic dimensions of body posture? An empirical evaluation of the comprehen- sive body examination. I. Nordic Journal of Psychiatry, 52, 319-326. doi:10.1080/08039489850149769

[6] Bunkan, B.H., Opjordsmoen, S., Moen, O., Ljunggren, A.E. and Friis, S. (1999) What are the basic dimensions of respiration? A psychometric evaluation of the comprehensive body examination. II. Nordic Journal of Psychiatry, 53, 361-369. doi:10.1080/080394899427836

[7] Breslau, N., Davis, G.C., Schultz, L.R. and Peterson, E.L. (1994) Migraine and major depression: A longitudinal study. Headache, 34, 387-393. doi:10.1111/j.1526-4610.1994.hed3407387.x

[8] Breslau, N., Lipton, R.B., Stewart, W.F., Schultz, L.R. and Welch, K.M.A. (2003) Comorbidity of migraine and depression: Investigating potential etiology and prognosis. Neurology, 60, 1308-1312.

[9] Benseñor, I.M., Tófoli, L.F. and Andrade, L. (2003) Headache complaints associated with psychiatric comorbidity in a population-based sample. Brazilian Journal of Medical and Biological Research, 36, 1425-1432. doi:10.1590/S0100-879X2003001000021

[10] Cao, M., Zhang, S., Wang, K., Wang, Y. and Wang, W. (2002) Personality traits in migraine and tension-type headaches: A five-factor model study. Psychopathology, 35, 254-258. doi:10.1159/000063829

[11] Zwart, J-A., Dyb, G., Hagen, K., Ødegård, K.J., Dahl, A.A., Bovim, G., et al. (2003) Depression and anxiety disorders associated with headache frequency. The NordTrøndelag health study. European Journal of Neurology, 10, 147-152. doi:10.1046/j.1468-1331.2003.00551.x

[12] Mongini, F., Ciccone, G., Deregibus. A., Ferrero, L. and Mongini, T. (2004) Muscle tenderness in different headache types and its relation to anxiety and depression. Pain, 112, 59-64. doi:10.1016/j.pain.2004.07.025

[13] Mongini, F., Deregibus, A. and Rota, E. (2005) Psychiatric disorders and muscle tenderness in episodic and chronic migraine. Expert Review of Neurotherapeutics, 5, 635-642. doi:10.1586/14737175.5.5.635

[14] Carleton, R,N., Abrams, M.P., Asmundson, G.J.G., Antony, M.M. and McCabe, R.E. (2009) Pain-related anxiety and anxiety sensitivity across anxiety and depressive disorders. Journal of Anxiety Disorders, 23, 791-798. doi:10.1016/j.janxdis.2009.03.003

[15] Carroll, L.J., Cassidy, J.D. and Côte, P. (2004) Depression as a risk factor for onset of an episode of troublesome neck and low back pain. Pain, 107, 134-139. doi:10.1016/j.pain.2003.10.009

[16] Jacobsen, L.N., Lassen, I.S., Friis, P., Videbech, P. and Licht, R.W. (2006) Bodily symptoms in moderate and severe depression. Nordic Journal of Psychiatry, 60, 294298. doi:10.1080/08039480600790358

[17] Cairns, D.M., Adkins, R.H. and Scott, M.D. (1996) Pain and depression in acute traumatic spinal cord injury: Origins of chronic problematic pain? Archives of Physical Medicine and Rehabilitation, 77, 329-335. doi:10.1016/S0003-9993(96)90079-9

[18] Kidd, R.F. and Nelson, R. (1993) Musculoskeletal dysfunction of the neck in migraine and tension headache. Headache, 33, 566-569. 
doi:10.1111/j.1526-4610.1993.hed3310566.x

[19] Vernon, H., Steiman, I. and Hagino, C. (1992) Cervicogenic dysfunction in muscle contraction headache and migraine: A descriptive study. Journal of Manipulative and Physiological Therapeutics, 15, 418-429.

[20] Gupta, R.K. (2009) Major depression: An illness with objective physical signs. World Journal of Biological Psychiatry, 10, 196-201. doi:10.1080/15622970902812072

[21] Matsui, T. and Fujimoto, T. (2011) Treatment for depression with chronic neck pain completely cured in $94.2 \%$ of patients following neck muscle treatment. Neuroscience \& Medicine, 2, 71-77.

[22] Fishbain, D.A, Goldberg, M., Rosomoff, R.S. and Roso- moff, H. (1991) Completed suicide in chronic pain. The Clinical Journal of Pain, 7, 29-36. doi:10.1097/00002508-199103000-00006

[23] Grant, D.M., Beck, J.G. and Davila, J. (2007) Dose anxiety sensitivity predict symptoms of panic, depression, and social anxiety? Behaviour Research and Therapy, 45, 2247-2255. doi:10.1016/j.brat.2007.02.008

[24] Andersen, J.H., Kaergaard, A., Frost, P., Thomsen, J.F., Bonde, J.P., Fallentin, N., et al. (2002) Physical, psychosocial, and individual risk factors for neck/shoulder pain with pressure tenderness in the muscles among workers performing monotonous, repetitive work. Spine, 27, 660667. doi:10.1097/00007632-200203150-00017 